\title{
A novel Nrf2 activator RS9 protects ARPE-19 cells from oxidative stress induced by $\mathrm{NaIO}_{3}$ via autophagic pathway
}

\author{
Yuichi Saito, Shinsuke Nakamura, Masamitsu Shimazawa, Hideaki Hara
}

Biofunctional Evaluation, Gifu Pharmaceutical University, Japan

\section{Background}

Non-exudative age-related macular degeneration (AMD) is the leading causes of severe loss of vision in the elderly. It is well known that the main symptom of this pathogenesis is retinal pigment epithelium (RPE) degeneration. Degenerated RPE lost their function for phagocytotic constitutive renewal of aged photoreceptor cells outer segment (POS). It directly leads to loss of visual function, which oxidative stress is at the bottom. A transcription factor which controls response of oxidative stress is Nrf2 (NF-E2-Related Factor2). Recently, a novel Nrf2 activator RS9 showed protective effects on light damaged mouse retina. However, closely mechanisms under Nrf2 activation and effects on RPE cells are not clear. To elucidate these points, we investigated the effects of RS9 against $\mathrm{NaIO}_{3}$ induced oxidative stress in ARPE-19 cells.

Methods

We used ARPE-19 cells, which is a human RPE cell line. ARPE-19 cells were seeded at 96 well plate. After 4 days incubation, oxidative stress was induced by $10 \mathrm{mM} \mathrm{NaIO}_{3}$ treatment. RS9 at $0.1-10 \mathrm{nM}$ were pretreated 6 hours prior to $\mathrm{NaIO}_{3}$ treatment. We assessed the contribution of autophagy pathway by using lysosomal acidification inhibitor chloroquine (CQ). Then, we evaluated cell viability, cell death rate, and mitochondrial membrane potential. The expression levels of autophagy marker LC3 and major anti oxidative protein heme oxygenase-1 (HO-1) were checked by western blot analysis. Additionally, we also evaluated RS9 effects in adult zebrafish (Danio rerio) light induced retinal degeneration model.

Results

RS9 treatment significantly improved cell viability and cell death rate after $\mathrm{NaIO}_{3}$ treatment. RS9 also improved mitochondrial activity which damaged by $\mathrm{NaIO}_{3}$ treatment. Moreover, protective effects of RS9 were abolished by $\mathrm{CQ}$ cotreatment. Consistent with these results, the up-regulation of LC3-II expression by RS9 treatment was abnormally further up-regulated by CQ co-treatment. This change indicated that CQ certainly stopped autophagic flux which is accelerated by RS9 treatment. On the other hand, HO-1 expression levels were up-regulated by RS9 treatment and not changed by CQ co-treatment. As well as in vitro assay, in zebrafish, CQ abolished protective effects of RS9.

Conclusions

We demonstrated that RS9 protected RPE cells from oxidative stress through autophagic pathway. 